\title{
Duality and Geometry Straightness, Characterization and Envelope
}

\author{
Jean-Marc Chassery ${ }^{1}$, David Coeurjolly ${ }^{2}$, and Isabelle Sivignon ${ }^{2}$ \\ ${ }^{1}$ Laboratoire LIS \\ Domaine universitaire Grenoble - BP46 \\ 38402 St Martin d'Hères Cedex, France \\ jean-marc. chassery@lis.inpg.fr \\ ${ }^{2}$ Laboratoire LIRIS - Université Claude Bernard Lyon 1 \\ Bâtiment Nautibus - 8, boulevard Niels Bohr \\ 69622 Villeurbanne cedex, France \\ \{david.coeurjolly, isabelle.sivignon\}@liris.cnrs.fr
}

\begin{abstract}
Duality applied to geometrical problems is widely used in many applications in computer vision or computational geometry. A classical example is the Hough Transform to detect linear structures in images. In this paper, we focus on two kinds of duality/polarity applied to geometrical problems: digital straightness detection and envelope computation.
\end{abstract}

\section{Introduction}

In domain of geometry, notion of duality is often used to represent the same structure in different domains like spatial domain or parametric one. The objective is to facilitate transformations like characterization, detection, recognition or classical ones such as intersection or union. A first example is illustrated with Voronoi partition in which polygonal regions are not homogeneous in terms of number of vertices. Nevertheless, the corresponding dual mesh, called Delaunay mesh, is composed of triangles. According to applications the choice of the alternative representations can be used on optimality criteria (computational cost, database structure, ...).

Following this first example, we focus in this paper on dual transformations illustrated by problems of digital straightness and envelope.

\section{Example of the Hough Transform}

The Hough transform (HT for short) is a very classical tool in image analysis to detect geometric features in images. These features may be line segments, circles, ellipses or any other parameterized curve. The HT, introduced in 1962 by Hough [1], is a dual transformation that enables to find a set of global structures, 
without any a priori knowledge on the number of structures to be found. Note also that this method is robust to noise and disconnected features.

\subsection{Definition of Hough Transform}

The general idea of this transform is that every point of the image contributes to the definition of the solution set for a given parameterized structure. Consider for instance a point $p_{0}$ of coordinates $\left(x_{0}, y_{0}\right)$ and the parameterization of lines $y=\alpha x+\beta$. Then the set of lines going through $p_{0}$ are the ones of parameters $(\alpha, \beta)$ fulfilling the equality $y_{0}=\alpha x_{0}+\beta$. This equality may be rewritten as $\beta=-\alpha x_{0}+y_{0}$, and if a new geometrical space $(\alpha \beta)$, called dual space, or parameter space, is defined, this equation defines a line : in this dual space, each point of this line represents a line of the $(x y)$ space going through the point $p_{0}$. An illustration of three points and the three corresponding lines in the dual space $(\alpha \beta)$ are represented in Figure 1 (a)-(b): note that the three lines in $(\alpha \beta)$ space are concurrent in one point, the coordinates of which defines a line going through the three points in $(x y)$ space.

However, as noticed by Duda in [2], the linear parameterization of lines defined by $y=\alpha x+\beta$ is not the handiest one since the two parameters $\alpha$ and $\beta$ are unbounded. Thus, another transform consists in using the polar parameterization of straight lines $\rho=x \cos \theta+y \sin \theta$. Any point in the $(x y)$ space defines a sinusoidal curve in the $(\theta \rho)$ space, where only the parameter $\rho$ has unbounded values (see Figure 1(c) for an illustration).

General properties fulfilled by these two representations, and suitable for straight line detection in images were expressed by Duda [2]:

\section{Property 1}

- A point in the $(x y)$ space matches up with one curve in the dual space;

- A point in the dual space matches up with a straight line in the $(x y)$ space;

- Points lying on a same line in the $(x y)$ space match up with concurrent curves in the dual space;

- Points on a same curve in the dual space match up with concurrent straight lines in the $(x y)$ space.

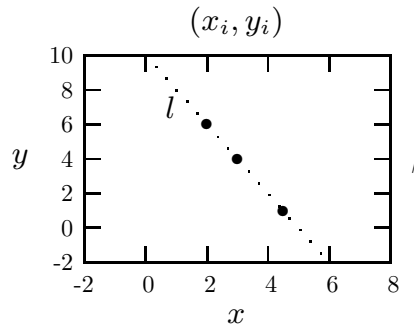

(a)

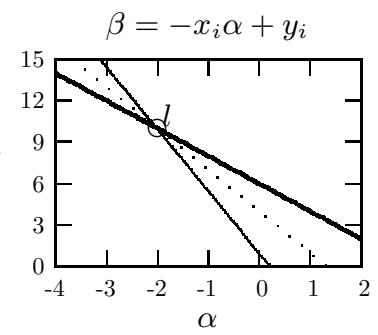

(b)

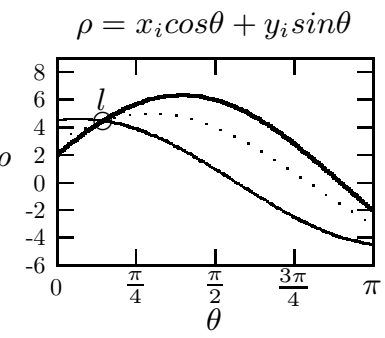

(c)

Fig. 1. (a) Three points in the $(x y)$ space; (b) Dual representation in the $(\alpha \beta)$ space; (c) Dual representation in the $(\theta \rho)$ space 


\subsection{Recognition of Parameterized Structures}

Line segment detection in images does not consist in finding the pixels lined up according to the Euclidean straight line definition, but a relaxation of this definition has to be used. To do so, the method generally used consists of, first, decomposing, or quantifying the dual space along the two axis, and second, defining a counter for each cell of the dual space. Algorithm 1 describes the general algorithm for finding parameterized curves in an image using HT. The quantization step is a trade-off between precision on one part, and memory/computation cost on the other hand. Moreover, a good quantization should provide constant densities for equally probable line parameters. An illustration of Algorithm 1 is proposed in Figure 2,

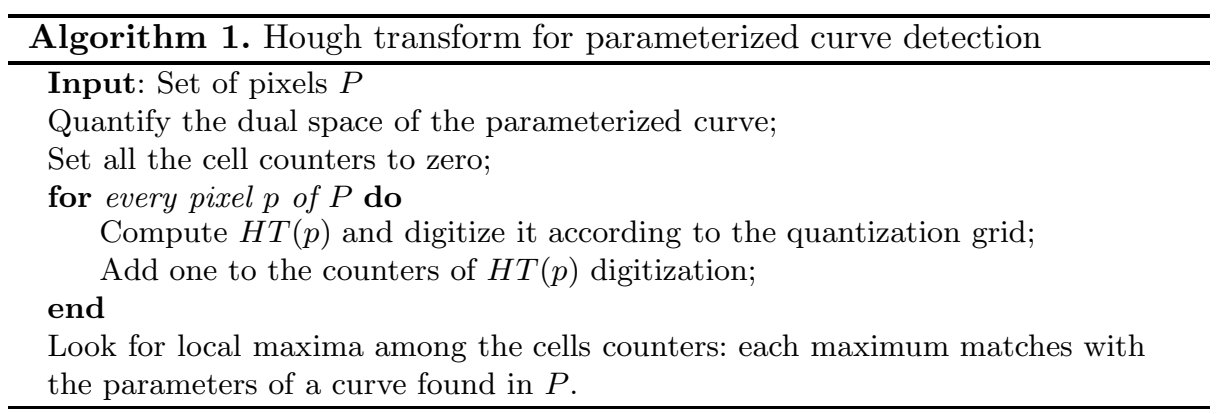

\section{Duality in Discrete Geometry}

During a HT, the discrete nature of the data processed is taken into account with a quantization of the dual space. On the contrary, we see in this section that the classical notion of dual space used in discrete geometry introduces the discrete nature of the data in the definition of the dual representation of a point.

\subsection{Definition of the Dual Space}

In digital geometry, pixels are said to be lined up if they belong to a digital straight line, which is the digitization of a straight line. In a general way, a digital straight line of parameters $(a, b, \mu)$ and bounds $\rho(a, b)$ and $\omega(a, b)$ is the set of pixels $(x, y)$ such that $\rho(a, b) \leq a x-b y+\mu \leq \omega(a, b)$. Without loss of generality, we suppose that $|b|>|a|$, and $b>0$ in the following. With these conditions, the previous definition may be rewritten as $\rho^{\prime}(\alpha, \beta) \leq \alpha x-y+\beta \leq \omega^{\prime}(\alpha, \beta)$. Given a point $p_{0}$ of coordinates $\left(x_{0}, y_{0}\right)$, the digital lines containing are the ones for which $\left(x_{0}, y_{0}\right)$ fulfills the inequalities. Thus, we can once again define a dual space $(\alpha \beta)$ to represent the space of line parameters, but contrary to HT, a given point $p_{0}$ of coordinates $\left(x_{0}, y_{0}\right)$ matches up with the intersection of two linear constraints defined by $E^{+}: \beta \geq-\alpha x_{0}+y_{0}+\rho^{\prime}(\alpha, \beta)$ and $E^{-}: \beta<-\alpha x_{0}+y_{0}+\omega^{\prime}(\alpha, \beta)$. 


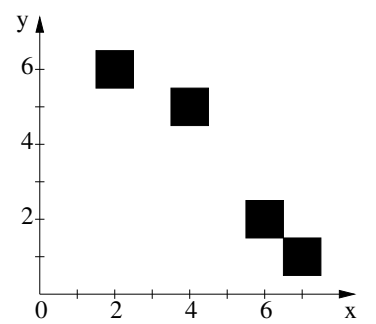

(a)

$$
\rho=x_{i} \cos \theta+y_{i} \sin \theta
$$

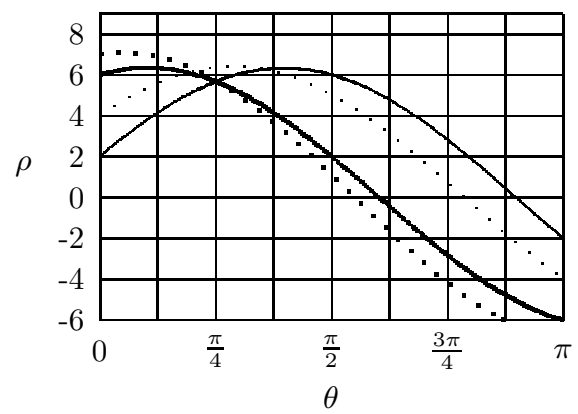

(b)

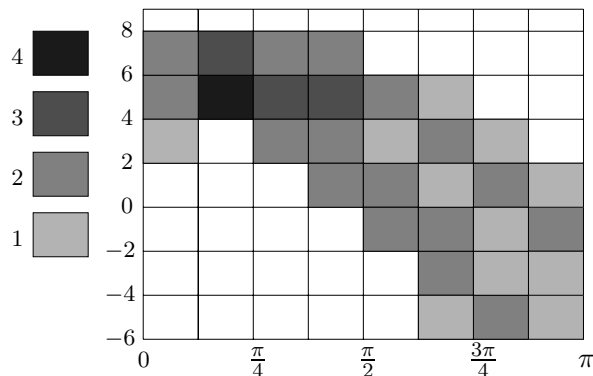

(c)

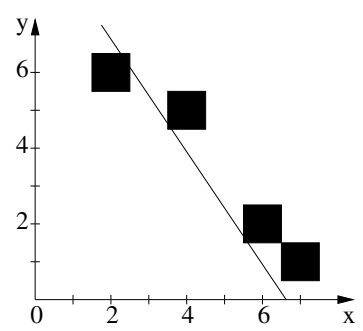

(d)

Fig. 2. Detection of a line segment with HT: (a) the four pixels of the set $P$; (b) dual representation in the quantified dual space; (c) result of the digitization of the sinusoidal curves; (d) straight line computed from the local maximum found

Definition 1. Let $P$ be a set of pixels. The preimage of $P$ denoted by $\mathcal{P}(P)$ is defined as follows: $\mathcal{P}(P)=\left\{(\alpha, \beta),|\alpha| \leq 1 \mid \forall(x, y) \in P, \rho^{\prime}(\alpha, \beta) \leq \alpha x-y+\beta<\right.$ $\left.\omega^{\prime}(\alpha, \beta)\right\}$. (See Figure 3 ).

As we can see, in digital geometry, the linear parameterization of lines is used in order to define the dual space. Nevertheless, we pointed out that for the Hough transform, using a polar parameterization is more convenient in order to handle bounded parameters. Actually, the polar parameterization is not appropriate for preimage definition since intersection of sinusoidal curves would be involved. Thus, the handling of unbounded domains has to be tackled. First, the parameter $\beta$ takes its values in an unbounded domain since it represents all the possible translation of a line. This problem is easy to solve, operating a translation of the set of pixels studied such that one particular pixel of the set is set to the origin. Next, the slope $\alpha$ of the lines also have unbounded values. The idea here is to use two dual spaces instead of one : 


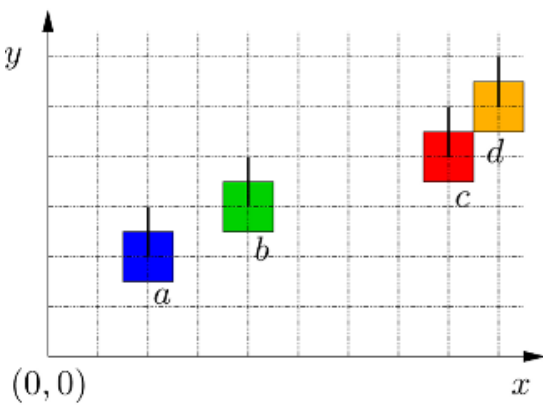

(a)

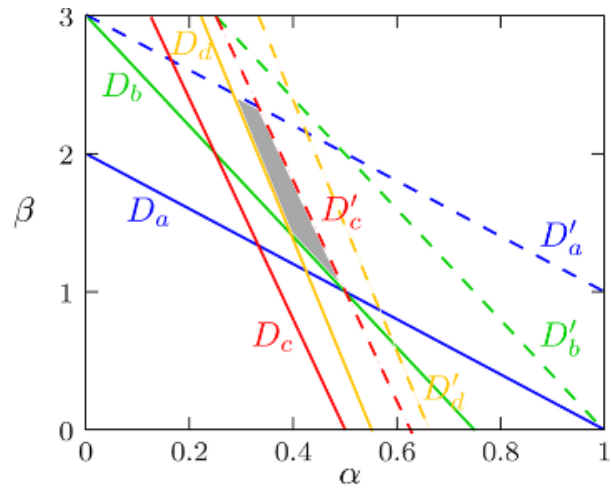

(b)

Fig. 3. Illustration of the preimage of a set of pixels (digitization process fixed): each point matches up with two linear constraints, and the preimage is the intersection of these constraints

Definition 2. The dual space $\mathcal{P}_{y}$ is defined as the space where one point $(\alpha, \beta)$, $|\alpha|<1$ stands for the line $\alpha x-y+\beta=0$. In the same way, a point $(\alpha, \beta)$, $|\alpha|<1$ of the dual space denoted $\mathcal{P}_{x}$ stands for the line $\alpha y-x+\beta=0$.

\subsection{Preimages of Digital Lines and Line Segments}

The definition of preimage depends on the values of $\rho^{\prime}(\alpha, \beta)$ and $\left.\omega^{\prime}(\alpha, \beta)\right\}$, and in most applications, these values are defined according to the digitization process considered during the definition of the digital straight line. In this section, firstly we give some examples of preimages of digital straight lines in respect to the digitization process considered, and secondly, we emphasize on particular properties of the preimage of digital straight line segments (DSS for short) for one digitization process.

Digitization and Preimage. Let us first consider the $O B Q$ (object boundary quantization) digitization scheme: given a straight line of equation $a x-b y+\mu=$ 0 , its $O B Q$ digitization is the set of pixels such that $0 \leq a x-b y+\mu<b$ (see conditions over $a$ and $b$ previously defined). Since the $O B Q$ digitization is based on the definition of the inside and this outside of an object, this definition assumes that the line $a x-b y+\mu=0$ is part of the boundary of an object the inside of which is given by the direction of the normal vector $(a,-b)$.

From this definition, we derive a characterization of the preimage of an infinite digital line according to the $O B Q$ digitization process [3]:

Property 2. Let $L$ be a digital straight line defined by $0 \leq a x-b y+\mu<b$, with $0 \leq a<b$. Then the preimage of $L$ according to the $O B Q$ digitization process is the vertical segment $\left[\left(\frac{a}{b}, \frac{\mu}{b}\right),\left(\frac{a}{b}, \frac{\mu+1}{b}\right)\right]$. 
Following a previous remark, this property assumes that the interior of object is "under" the line (see Figure 4 left). Otherwise the preimage of $L$ is the segment $\left[\left(\frac{a}{b}, \frac{\mu+1}{b}-1\right),\left(\frac{a}{b}, \frac{\mu}{b}-1\right)[\right.$ (see Figure 4, right).

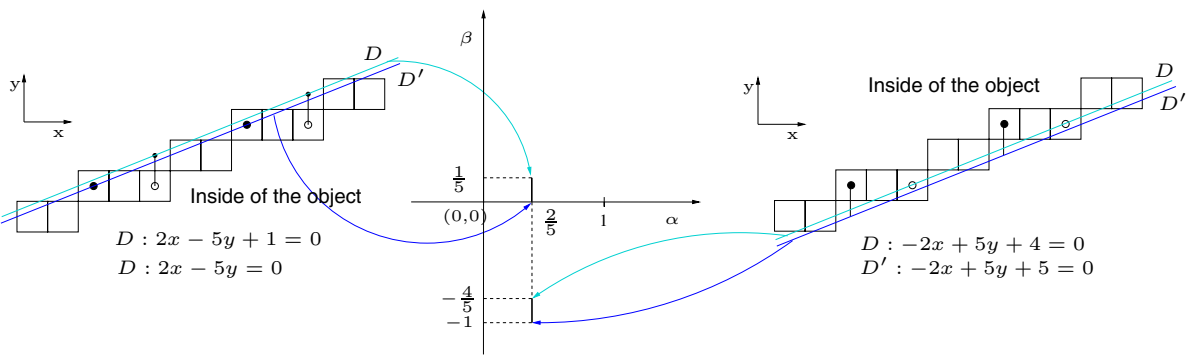

Fig. 4. Preimage of the digital straight line $0 \leq 2 x-5 y<5$ : two solutions according to the direction of the solutions normal vector

Let us now consider the standard digitization process: given a straight line of equation $a x-b y+\mu=0$ such that $0 \leq a<b$, its standard digitization is the set of pixels such that $-\frac{|a|+|b|}{2} \leq a x-b y+\mu<\frac{|a|+|b|}{2}$. Contrary to the $O B Q$ digitization process, the standard digitization of a line does not depend on the direction of the normal vector of the line. However, we have the same kind of results on the characterization of the preimage (see Figure 5 for an illustration):

Property 3. Let $L$ be a digital straight line defined by $-\frac{|a|+|b|}{2} \leq a x-b y+\mu<$ $\frac{|a|+|b|}{2}$, with $0 \leq a<b$. Then the preimage of $L$ according to the standard digitization process is the vertical segment defined by:

$-\left[\left(\frac{a}{b}, \frac{\mu}{b}\right),\left(\frac{a}{b}, \frac{\mu+1}{b}\right)[\right.$ if $|a|+|b|$ is even;

$-\left[\left(\frac{a}{b}, \frac{\mu}{b}-\frac{1}{2 b}\right),\left(\frac{a}{b}, \frac{\mu}{b}+\frac{1}{2 b}\right)[\right.$ if $|a|+|b|$ is odd.

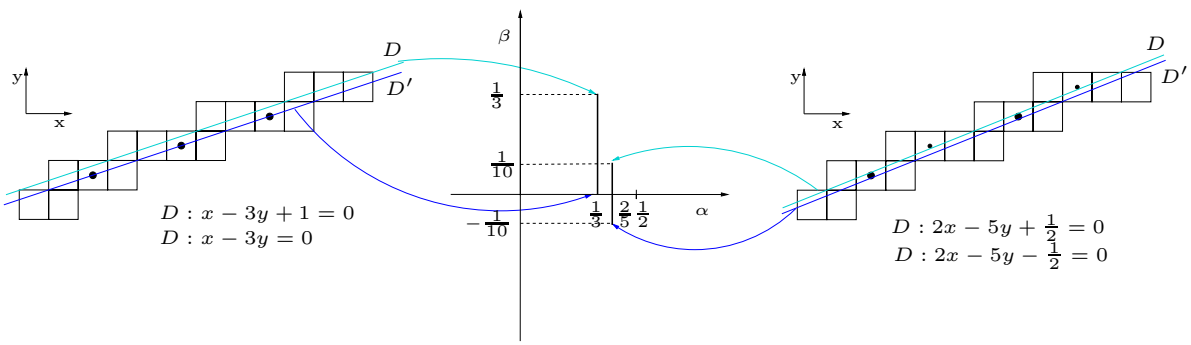

Fig. 5. Preimages of digital straight lines according to the standard digitization process: on the left, the sum $|a|+|b|$ is even, on the right, it is odd 
DSS Preimage. Using a dual space is a common technique for digital straight line recognition, and thus many works have been carried out about the geometrical and arithmetical structure of the preimage of a digital straight segment. We simply recall here some of the main and classical properties on this structure 4, 5]:

Property 4. Let $S$ be a set of $N+18$-connected pixels, and $x_{0}$ be the minimum abscissa of this set of pixels. Then the preimage $\mathcal{P}(S)$ of $S$ has the following properties:

1. $\mathcal{P}(S)$ is a convex polygon with at most four vertices;

2. two consecutive abscissa of the vertices are consecutive terms in the Farey series [6] of order $\max \left(x_{0}, N-x_{0}\right)$. Moreover, for a given abscissa equal to $\frac{p}{q}$, the corresponding ordinate is a multiple of $\frac{1}{q}$;

3. if this polygon has four vertices, then two out of the four vertices have the same abscissa.

This property shows that there is a strong connection between DSS preimages and Farey series. Actually, given a Farey series of order $d$, a Farey diagram may be defined ( 5], see Figure 6] on the left for an example). An important property of this diagram is that their is a bijection between the cells of the Farey diagram of order $q$ and the preimages of the DSS of length $q+1$ [7. This property is illustrated in Figure 6 in the case of $q=2$ : there are only four DSS of length 3, and their preimages are the cells of the diagram. These strong arithmetical and geometrical features of DSS preimages are used to design efficient recognition algorithms 8,9].

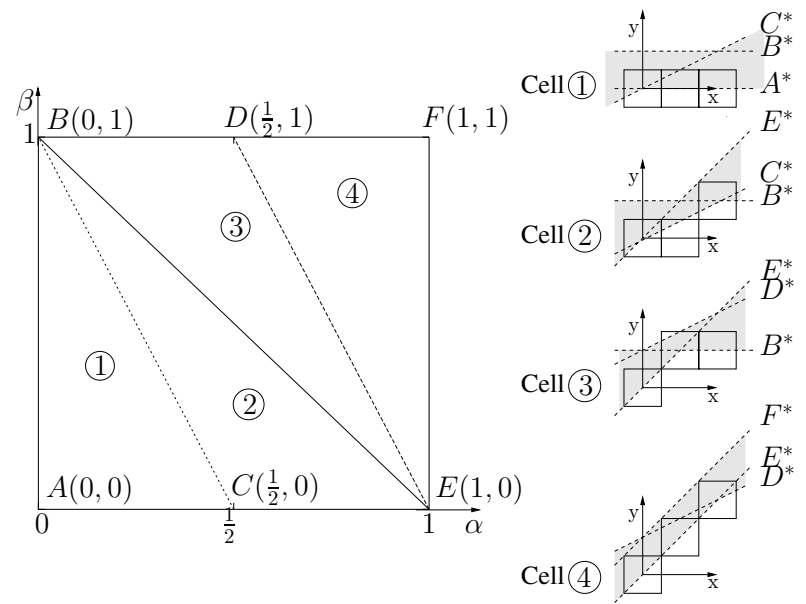

Fig. 6. Bijection between the cells of a Farey diagram of order 2 and the preimages of DSS of length 3 


\section{Generalizations and Applications}

These definitions of dual space and preimage easily extend to higher dimensions for digital hyperplanes. Nevertheless, even if the characterization of the preimages of infinite hyperplanes is easy to handle, few works have been carried out concerning the structure of the preimage in $3 \mathrm{D}$ or more. In [10], the authors propose a first structural and arithmetical characterization of the preimage of a digital plane segment for particular cases. In [11], a theoretical and experimental study on the number of faces of the preimage in higher dimensions is proposed. Concerning the definition of dual space and preimage, recent work 12 proposes a generalization of the preimage which enables to define the dual of a polygon as the set of lines crossing this polygon. Together with the use of standard digital planes, this new dual structure enables to define nice algorithms for digital curve reconstruction.

Concerning the interest of using a dual space for applications, we already mentioned the digital line recognition problem, for which using a dual space offers a nice solution. This algorithm can also be extended for digital plane segments recognition [13. This notion may also be used to study the properties of the intersection of two digital lines or two digital planes, as in [14. In this case, the preimage of infinite digital lines and planes are involved in the characterization of the minimal parameters of the set of intersection grid points. Finally, let us also mention the work carried out by Veelaert in [15, 16, 17, concerning the detection of collinear, parallel or concurrent segments in an image. In these works, the dual representation of the studied properties enables to extract a graph in which particular structures (e.g. cliques) are sought. Then these structures represent sets of segments fulfilling the desired property.

\section{Duality/Polarity and Convexity}

\subsection{Definitions}

Another way to consider the geometrical duality is to consider the projective group on $(d+1)$ homogeneous coordinates. The homogeneous representation of a point $\boldsymbol{p}=\left(x_{1}, \ldots, x_{d}\right)$ in the $d$-dimensional Euclidean space is the point $\left(x_{1}, \ldots, x_{d}, 1\right)$ in the projective space [18]. Furthermore, for any non-zero scalar $\lambda$, the homogeneous points $\left(\lambda x_{1}, \ldots, \lambda x_{d}, \lambda\right)$ represent the same point in the Euclidean space.

This representation framework is convenient to obtain a matrix representation of both affine transformations and duality mappings. Indeed, with $(d+1)$ homogeneous coordinates, we can represent Euclidean points and all linear varieties of dimension $k<d+1$. To define the polarity, we consider the following transformation:

$$
\boldsymbol{\alpha}=\boldsymbol{\beta} B
$$

where $\boldsymbol{\alpha}$ and $\boldsymbol{\beta}$ are vectors in $(d+1)$ homogeneous coordinates and $B$ is a $(d+1) \times(d+1)$ matrix. 
In the following, we focus on matrices $B$ such that $|B| \neq 0$ (defining the projective group) and $B=B^{T}$. In dimension 2 , this transformation maps points to lines and lines to points using the homogeneous coordinate system. Furthermore, this class of transformation preserves the incidence: $\boldsymbol{\alpha} \in \boldsymbol{\beta}$, then $\boldsymbol{\beta} B \in \boldsymbol{\alpha} B$. We thus obtain a geometric duality.

Note that the matrix $B$ is such that $\boldsymbol{\alpha} B \boldsymbol{\alpha}^{T}=0$ which corresponds to the equation of a conic in homogeneous coordinates [18. In computational geometry, the mapping induced by such a conic is called polarity.

\subsection{Polarity with Respect to Unit Circle}

In the following, we focus on the polarity defined by the matrix:

$$
B=\left[\begin{array}{ccc}
1 & 0 & 0 \\
0 & 1 & 0 \\
0 & 0 & -1
\end{array}\right]
$$

The conic defined by this mapping in homogeneous coordinates is a unit circle in dimension 2. This transformation maps a point $\left(x_{1}, x_{2}, 1\right)$ to the line $x_{1} x+$ $x_{2} y-z=0$ in homogeneous coordinates.

A final property of this special duality is that we have a kind of metric preservation. Given a point (or a line) $\boldsymbol{\alpha}$, we have:

$$
\operatorname{distance}(\boldsymbol{\alpha}, 0) \cdot \operatorname{distance}(\operatorname{dual}(\boldsymbol{\alpha}), 0)=1
$$

In the Cartesian space, we can thus consider the dual by polarity of a polygon. Note that the center of the unit circle (called the pole) needs to be specified. Figure 7 illustrates the dual transformation of each straight line defined by the polygon edges. Connecting the polar elements using the incidence property, we thus obtain a polygon (not necessarily simple) called the dual polygon.

Using the classical property of polarity that maps union to intersection and conversely we mention the property that in the general case, the convex hull
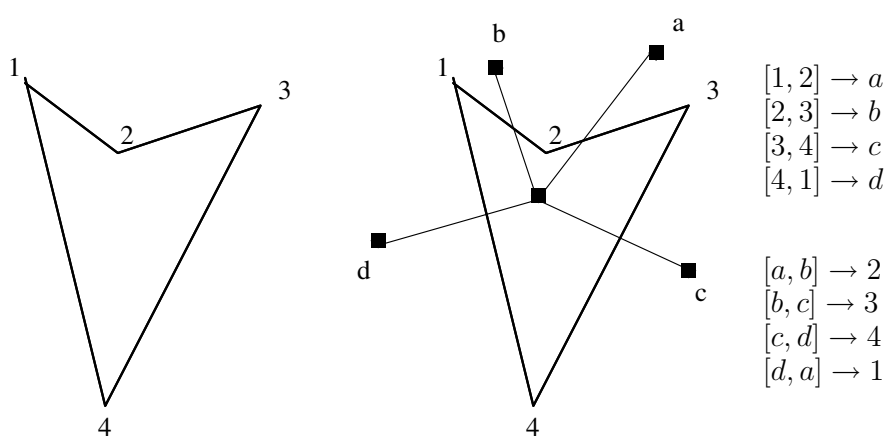

Fig. 7. A polygon and its dual by polarity 

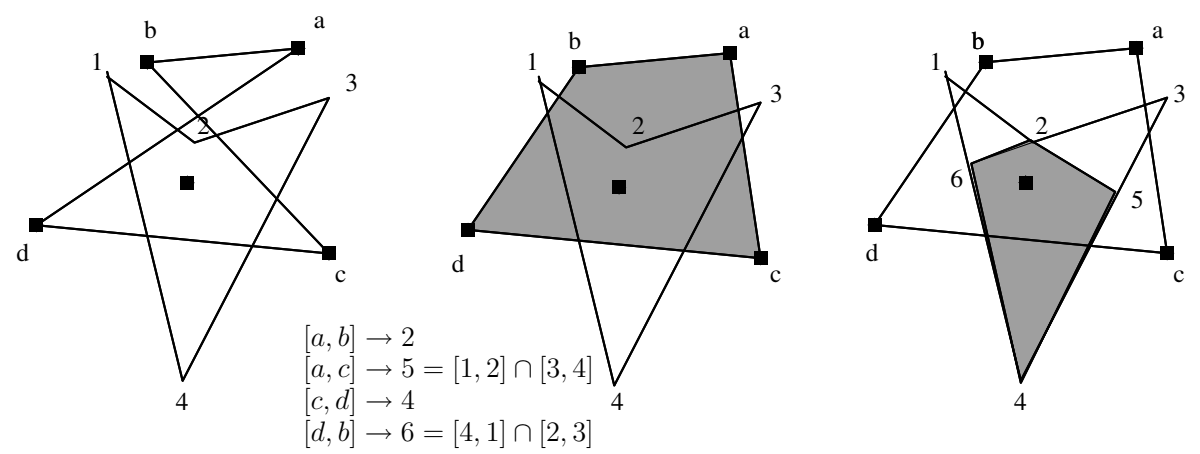

$\operatorname{dual}[1,2,3,4]=[a, b, c, d]$

$\operatorname{dual}[a, b, d, c]=[2,6,4,5]=\operatorname{Kern}[1,2,3,4]$

Fig. 8. A polygon, its kernel as the dual of the convex hull of the dual

of the dual polygon corresponds to the dual of a geometrical object called the kernel. This object corresponds to the set of all the points inside the polygon which are visible from any point of polygon contour (see below for a formal definition of the kernel). The class of polygons the kernel of which is not empty is called the star-shaped class of polygons. Figure 8 illustrates this construction (remind that the position of the pole is very important).

\section{Application to Convex Optimization by Interior}

This section addresses the problem of the access of the shape by its interior. Polygon inclusion problems are defined as follows: given a non-convex polygon, how to extract the maximum area subset included in that polygon ? The search of the maximum horizontal-vertical convex polygon included into a simple orthogonal polygon has been solved in the continuous case by Wood and Yap with complexity in $O\left(n^{2}\right)$ [19]. For more details about a lot of proposed solutions to inclusion problems, refer to 20 .

For the rest of the presentation, we consider a polygon $P=\left(v_{0}, v_{1}, \ldots, v_{n-1}\right)$ with $n$ vertices. We denote by $R=\left(r_{0}, r_{1}, \ldots, r_{k-1}\right)$ the $k$ reflex vertices (or concave vertices) of $P$ (maybe empty). We note by $C_{i}$ a chord supported by two successive vertices $v_{i}$ and $v_{i+1}$. The potato-peeling problem can be expressed as follows: Find the maximum area convex subset (MACS for short) $Q$ contained in $P$.

In [21], Goodman proves that $Q$ is a convex polygon. He presents explicit solutions for $n \leq 5$ and leaves the problem unsolved in the general case.

In 22], Chang and Yap prove that the potato-peeling problem can be solved in polynomial time in the general case. More precisely, they detail an $O\left(n^{7}\right)$ time algorithm to extract $Q$ from $P$. Since this algorithm uses complex geometric concepts and dynamic programming in several key steps, it is not tractable in practical applications. 
In the following we propose an approximation based algorithm to approach the MACS of a star-shaped polygon $P$. The proposed algorithm is an iterative process based on a kernel dilatation framework.

\subsection{Fast Approximation Algorithm}

In this section, we assume that $P$ is a star-shaped polygon. $P$ is a star-shaped polygon if there exist a point $q$ in $P$ such that $q \bar{v}_{i}$ lies inside $P$ for all vertices $v_{i}$ of $P$. The set of points $q$ satisfying this property is called the kernel of $P$.

An extremal chord is a chord which contains two or more vertices of $\mathrm{P}$. We note that an edge of $\mathrm{P}$ is always included in an extremal chord.

To end with definitions a chord is called single-pivot chord if it contains only one reflex vertex (chord $C_{1}$ in Figure 9) and double-pivot chord if it contains two distinct reflex points (chord $C_{2}$ in Figure 9).

The kernel of $P$ can be seen as the intersection between $P$ and the halfplanes $C_{i}^{+}$defined by all extremal chords $C_{i}$ associated to all reflex vertices, as illustrated in Figure 10.

Figure 10 is an illustration of such proposition. We have the property:

Property 5. Let $P$ be a star-shaped polygon, then its kernel is a subset of the maximum area convex subset of $P$.

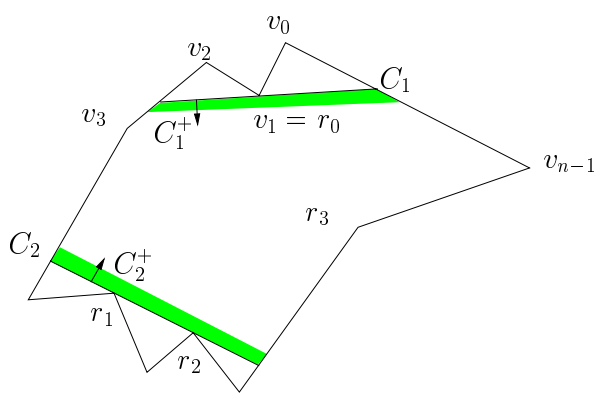

Fig. 9. Notations and illustrations of chords and half-planes generated by these chords

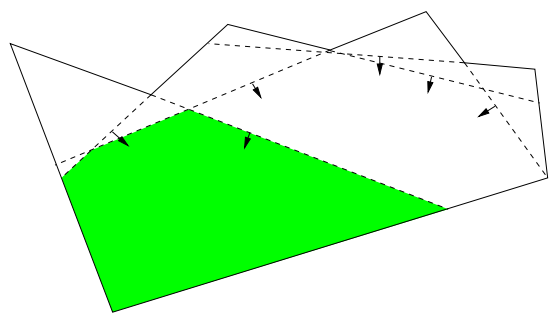

Fig. 10. Illustration of the kernel computation based on intersection of extremal chords 
Proof. Details of the proof are given in [20].

In other words, there exists a continuous deformation that transforms the kernel to the MACS. In the following, the strategy we choose to approximate the MACS is to consider the deformation as an Euclidean dilatation of the kernel. Based on this heuristic, several observations can be made: the reflex vertices must be taken into account in the order in which they are reached by the dilatation wavefront. More formally, we consider the list $\mathcal{O}$ of reflex vertices such that the points are sorted according to their minimum distance to the kernel polygon. When a reflex vertex is analyzed, we fix the possible chords as follows:

- the chord may be an extremal one;

- the chord may be a single-pivot chord such that its slope is tangent to the wavefront (this point will be detailed in the next section);

- the chord may be a double-pivot chord. In that case, the second reflex vertex that belongs to the chord is necessary. It must correspond to the next reflex point in the order $\mathcal{O}$.

Furthermore, when a reflex vertex is analyzed, we choose the chord from this list that maximizes the area of the resulting polygon. If we denote by $P^{\prime}$ the polygon given by the intersection between $P$ and the half-plane associated to the chosen chord, the chord must maximize the area of $P^{\prime}$. In the algorithm, it is equivalent to minimize the area of the removed parts $P / P^{\prime}$. Using these heuristics, the approximated MACS algorithm can be easily designed in a greedy process.

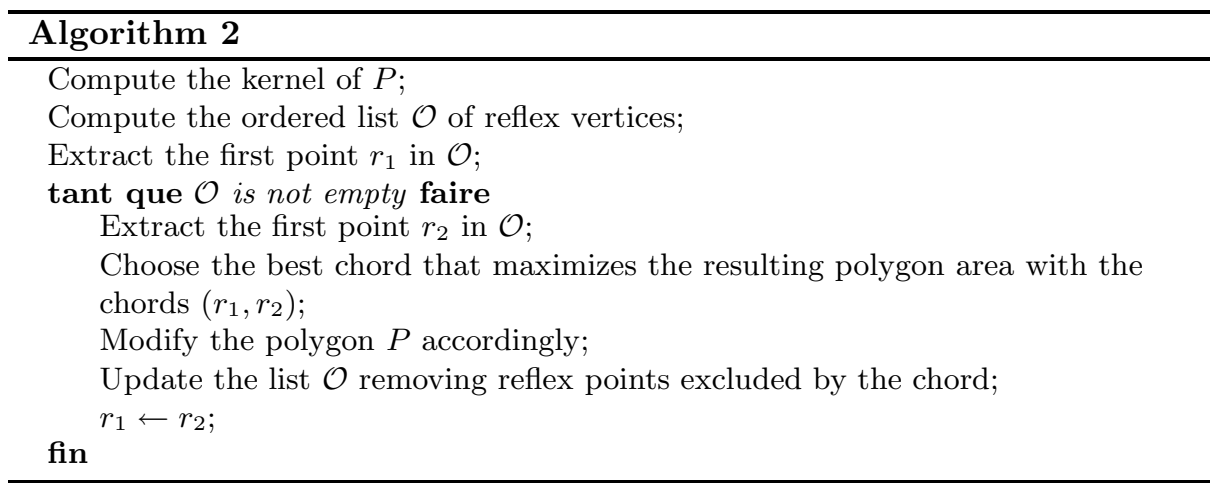

\subsection{Single-Pivot Chords Computation}

Given a reflex point $r_{i}$ of $P$, we have listed three possible classes of chord: extremal, single-pivot and double-pivot chords. The Figure 11 reminds the possibles chords. The extremal and double-pivot chord computation is direct. However, we have to detail the single-pivot chord extraction. According to our heuristic, the single-pivot chord associated to $r_{i}$ must be tangent to the wavefront propagation of the kernel dilatation. 

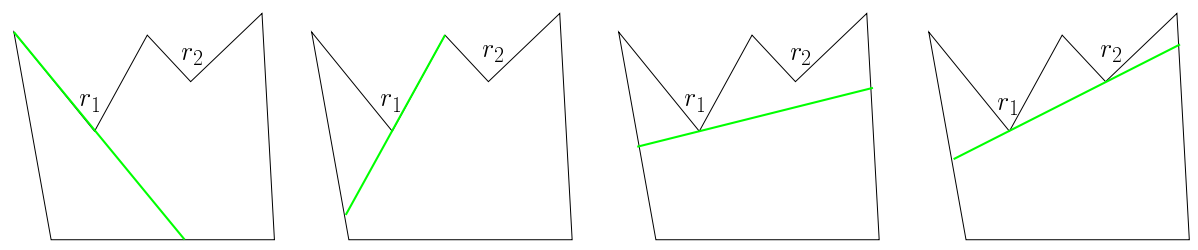

Fig. 11. All possible chords that can be associated to the reflex point $r_{1}$ (the two extremal chords, a single-pivot balanced chord and the double-pivot chord)

Using the exterior angular bisector structure issued from the computation of the generalized Voronoi diagram of $\operatorname{Kern}(P)$, we can efficiently compute the slopes of such chords. In Figure 12, let $e_{1}$ and $e_{2}$ be two adjacent edges of $\operatorname{Kern}(P)\left(e_{1}\right.$ and $e_{2}$ are incident to the vertex $\left.v\right)$. Let $p$ (resp. $q$ ) be a point in the plane that belongs to the cell generated by $e_{1}$ (resp. $e_{2}$ ). We can distinguish two cases: $p$ is closer to $e_{1}$ than to one of its extremities and $q$ is closer to $v$ than to $e_{2}$ (without the extremities). Hence the straight line going through $p$ and tangent to the wave-front propagation is parallel to $e_{1}$. In the second case, the tangent to wavefront straight line going through $q$ is tangent to the circle of center $v$ and radius $\|\boldsymbol{v} \boldsymbol{q}\|$ (see Figure 12).

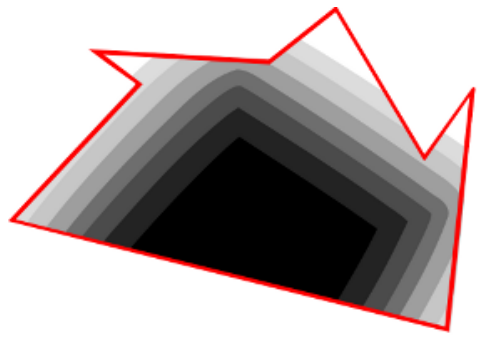

(a)

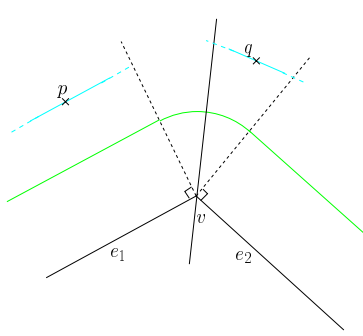

(b)

Fig. 12. Computing a chord parallel to the kernel dilatation wavefront: (a) illustration of the kernel dilatation, (b) single-pivot slope computation

Finally, if each reflex point $r_{i}$ of $P$ is labelled according to the closest edge $e_{i}$ of $\operatorname{Kern}(P)$ (extremities included), we can directly compute the single-pivot chord: if $r_{i}$ is closer to $e_{j}$ than one of its extremities, the chord is parallel to $e_{j}$, otherwise, the chord is tangent to a given circle. Computational cost analysis is developed in details in [20].

\subsection{Experiments}

In this section, we present some results of the proposed algorithm. First of all, Figure 13 compares the results between the optimal Chang and Yap's algorithm 22] and the approximated MACS extraction process on 3 examples. In 

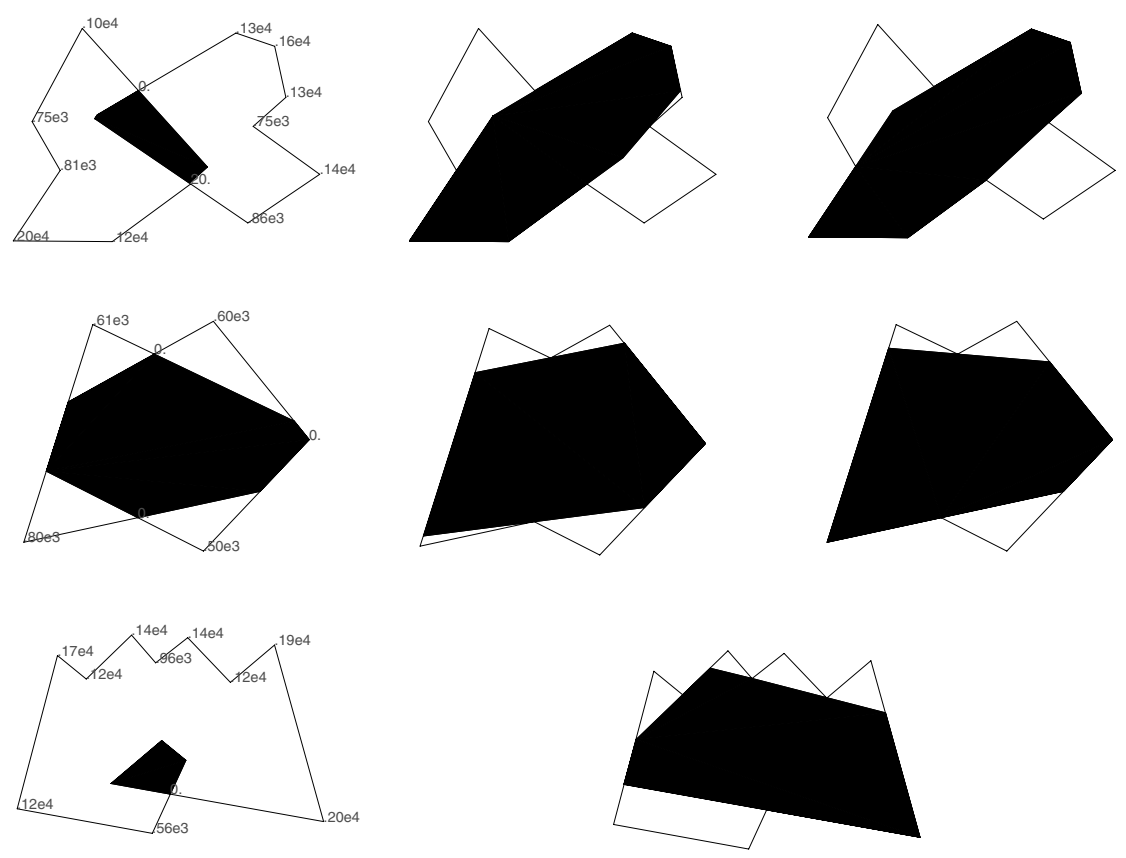

Fig. 13. Comparisons between the optimal MACS and the fast approximation proposed algorithm. Each line corresponds to a new example. The first column presents the input polygons, their kernels and the distance labelling, the second column shows the results of the Chang and Yap's algorithm. The last column presents the result of the proposed algorithm. For the third example, optimal MACS and the fast approximation proposed algorithm offer the same result.

practical experiments, the optimal $O\left(n^{7}\right)$ algorithm do not lead to a direct implementation. Indeed, many complex geometrical concepts are used and the overall algorithm is not really tractable. In Figure 13, the first column presents the polygon, its kernel and the distance labelling of all vertices, the second row contains the optimal MACS and the third one the fast approximation of the MACS presented in Section 5.1. Note that the results of the last row are identical. If we compute the area error between the optimal and the approximated MACS on these examples, the error is less than one percent.

\section{Conclusion}

Duality can be seen in two ways : a geometric or a parametric transform. Geometrical duality and graphs have been widely investigated with Voronoi diagrams and Delaunay triangulations. In this paper, we focused on transformation based duality. Two different processes have been investigated. The first one is based on characterization of digital straight lines using duality and preimage. The second 
one is based on polarity for kernel construction used as an initialization step for the problem of convex envelope of polygon by interior. The analysis of the geometry of the dual polygon seems to be very promising to have a direct computation of the MACS. Furthermore, a unification of both duality and polarity frameworks is a challenging future work. Many other examples or approaches could be studied and elaborated using this concept of duality. All these methods share the choice of alternative representations in order to offer optimality criteria.

\section{References}

1. Hough, P.: Method and means for recognizing complex patterns. United States Patent, n3, 069, 654 (1962)

2. Duda, R.O., Hart, P.E.: Use of the Hough transformation to detect lines and curves in pictures. Communications of the ACM 15(1) (1972) 11-15

3. Sivignon, I.: De la caractérisation des primitives la reconstruction polyédrique de surfaces en géométrie discrète. Thèse de doctorat, Institut National Polytechnique de Grenoble, Grenoble, France (2004)

4. Dorst, L., Smeulders, A.N.M.: Discrete representation of straight lines. IEEE Trans. on Pattern Anal. and Mach. Intell. 6(4) (1984) 450-463

5. McIlroy, M.D.: A note on discrete representation of lines. AT\&T Technical Journal 64(2) (1985) 481-490

6. Hardy, G.H., Wright, E.M.: An Introduction to the Theory of Numbers. Oxford Society (1989)

7. Vittone, J.: Caractérisation et reconnaissance de droites et de plans en géométrie discrète. Thèse de doctorat, Université Joseph Fourier, Grenoble, France (1999)

8. Lindenbaum, M., Bruckstein, A.: On recursive, $\mathcal{O}(n)$ partitioning of a digitized curve into digital straight segments. IEEE Trans. on Pattern Anal. and Mach. Intell. 15(9) (1993) 949-953

9. Coeurjolly, D.: Algorithmique et géométrie discrte pour la caractrisation des courbes et surfaces. Thèse de doctorat, Université Lumière Lyon 2, Lyon, France (2002)

10. Coeurjolly, D., Sivignon, I., Dupont, F., Feschet, F., Chassery, J.M.: On digital plane preimage structure. Discrete Applied Math. 151(1-3) (2005) 78-92

11. Coeurjolly, D., Brimkov, V.: Computational aspects of digital plane and hyperplane recognition. In: 11th International Workshop on Combinatorial Image Analysis. LNCS, Springer-Verlag (2006)

12. Dexet, M., Andres, E.: Linear discrete line recognition and reconstruction based on a generalized preimage. In Reulke, R., Eckhardt, U., B., F., U., K., Polthier, K., eds.: 11th International Workshop on Combinatorial Image Analysis. Volume 4040 of LNCS., Springer-Verlag (2006) 174-188

13. Vittone, J., Chassery, J.M.: Recognition of digital naive planes and polyhedrization. In Borgefors, G., Nystrm, I., Sanniti di Baja, G., eds.: Discrete Geometry for Computer Imagery. Volume 1953 of Lect. Notes on Comp. Sci., Springer-Verlag (2000) 296-307

14. Sivignon, I., Dupont, F., Chassery, J.M.: Digital intersections : minimal carrier, connectiviy and periodicity properties. Graphical Models 66(4) (2004) 226-244

15. Veelaert, P.: Geometric constructions in the digital plane. Journal of Mathematical Imaging and Vision 11 (1999) 99-118 
16. Veelaert, P.: Collinearity and weak collinearity in the digital plane. In Bertrand, G., Imiya, A., Klette, R., eds.: Digital and Image Geometry. Volume 2243 of Lect. Notes on Comp. Sci., Dagstuhl, Allemagne, Springer-Verlag (2001) 439-453

17. Veelaert, P.: Graph-theoretical properties of parallelism in the digital plane. Discrete Applied Mathematics 125 (2003) 135-160

18. Preparata, F.P., Shamos, M.I.: Computational Geometry : An Introduction. Springer-Verlag (1985)

19. Wood, D., Yap, C.K.: The orthogonal convex skull problem. Discrete and Computational Geometry 3 (1988) 349-365

20. Chassery, J.M., Coeurjolly, D.: Optimal shape and inclusion: open problems. In Ronse, C., Najman, L., Decencière, E., eds.: International Symposium on Mathematical Morphology. Volume 30 of Computational Imaging and Vision., Springer (2005) 229-248

21. Goodman, J.E.: On the largest convex polygon contained in a non-convex $n$-gon or how to peel a potato. Geometricae Dedicata 11 (1981) 99-106

22. Chang, J.S., Yap, C.K.: A polynomial solution for the potato-peeling problem. Discrete \& Computational Geometry 1 (1986) 155-182 